\title{
Affordance Based Assessment of Ligament \\ Healing in the Knee Joint
}

International

Journal of

Orthopedics and

Sports Medicine

(IJOSM)

\section{Volume 2 Issue 1, 2021}

\section{Article Information}

Received date : February 09, 2021

Published date: February 27, 2021

\section{*Corresponding author}

Wangdo Kim, Ingeniería Mecánica,

Universidad de Ingenieria y Tecnologia, Lima, Perú

\section{Keywords}

Ecological Biomechanics; KneeTensegrity-Structure (KTS); AffordanceBased-Assessment; Knee Synergy; Entrainment; Instantaneous knee Screw (IKS); Virtual Motion

Distributed under: Creative Commons CC-BY 4.0

\author{
Wangdo Kim ${ }^{1 *}$ and Sang "_"Gook Kim ${ }^{2}$ \\ ${ }^{1}$ Ingeniería Mecánica, Universidad de Ingenieria y Tecnologia-UTEC, Lima, Perú \\ ${ }^{2}$ Mechanical Engineering, Massachusetts Institute of Technology (MIT), Cambridge, MA, USA
}

\section{Abstract}

There is increasing awareness of the need to include patient-reported outcome (PRO) instruments in the evaluation of ligament healing in the knee Joint. Surgical design in personalized medicine is often based on native anatomy, which may not accurately reflect the sensory function of the joint structure, including native musculoskeletal tissues and biomechanical artifacts. To overcome this problem, researchers have developed alternative approaches based on affordance-based assessment. Estimating the instantaneous knee screw (IKS) axis is crucial to implanting a prosthesis and accessing joint kinematics. The correct estimation of the patient-specific joint axis of the knee is essential for achieving a reliable assessment of musculoskeletal kinematics and dynamics. We found that active touch and posture refer to what is ordinarily called touching-variations in skin stimulation caused by surfaces are altered together by motor activity variations. This affordance of "walk-on-able" is worth noting because it is often neglected that locomotion and its surfaces form an inseparable pair. The assessment process can be viewed in terms of action possibilities provided by the active sets of organs residing that can obtain and utilize information about the tissue environments in which the grafts are to be located.

Anterior Crucial Ligament Reconstruction and its Assessment

The anterior cruciate ligament (ACL) is a critical knee joint, bone-to-bone connected, stability ligament that is attached from an anterior location of the proximal tibia to a posterior location of the distal femur. The ACL is highly susceptible to failure during athletic activities and slip-fall events [1]. The goal of ACL reconstruction surgery is to rebuild the ligament attachments as closely as possible to the native anatomy in order to restore pre-injury knee function and normal proprioception in the affected knee [2]. Personalized medicine in surgery allows the customization of insertion sites, graft size, tunnel placement, and graft tension for each individual patient [3]. A critical pre-operative decision concerns the placement of a tibial-femoral tunnel mimicking the native orientation of the ACL attachment [3]. Surgeons need to consider particular aspects of the local anatomy and, by extension, the biomechanical artifacts introduced during surgery.

Considering the importance of the sensory function of the joint structure, it would seem sensible to minimize the sensory damage of the joint whenever operative treatment is necessary [4]. The joints are exploratory sense organs, but they are also performatory motor organs; that is to say, the equipment for feeling is anatomically the same as the equipment for doing [5]. Here, we report an alternative approach based on the understanding of knee affordances to guide surgeons in the design/assessment of knee reconstruction strategies. To our knowledge, this is the first study to use psychological theory to address this surgical assessment concept [6]. Traditional rating systems to assess clinical outcome after joint arthroplasty are often based on the surgeon's objective ratings, such as range of motion and strength, or clinical ratings of function and pain. However, the patient's perceptions after arthroplasty may differ significantly from those of their clinician. Moreover, surgeons often underappreciate the needs and views of their patients [7].

There is, therefore, increasing awareness of the need to include patient-reported outcome (PRO) instruments in the evaluation of surgical procedures. Indeed, these patient-centered assessments of treatment outcomes are becoming today's standard [8]. Patient-reported outcome metrics (PROMs) can be simply described as a patient's health status self-report. A forgotten joint score, corresponding to when a patient forgets the artifact in their everyday life, was introduced in PROM as the ultimate goal in joint reconstruction [9]. 'Forgotten joint scores' are often observed in patients after surgery [10]. Nevertheless, these ratings do not replace the need to understand the general role of artifacts and affordances in reconstruction surgery. This study aims to identify measurable invariants using a (positive) affordance-based assessment strategy for the structural function of the joint during ACL reconstruction. The term 'affordance' is conventionally traced to James J. Gibson, and his programmatic approach to perception and action, Ecological Psychology [11]. The notion appears simple at its core, and yet upon closer examination, it has the potential to reveal a radically altered view of the relation between an organism and its environment [12]. The fundamental hypothesis of the ecological approach and this work is that active organisms of the knee that can obtain and utilize information about persisting properties of their tissue environments in which the grafts are to be located will have a definite advantage over organisms that cannot do this.

\section{Affordance of the knee}

Gibson demonstrated how animal perception and action is continuous, with interactions with inanimate objects or surfaces [11]. The affordances of a product are what it provides, offers, or furnishes to a user. Gibson's 'system theory' of perception corresponds to an open system, which is rather different from the view of isolated artifacts [5]. The resources encountered by an animal or thinking humans are the affordances of the environment. Affordances are opportunities for action, not causes or stimuli [13]. The impetus for any knee surgery project can be understood in terms of creating and changing affordances. The design process is the construction of an artifact that offers specific affordances but not certain undesired affordances. An artifact with more positive affordances is considered better, while an artifact with more negative affordances is considered worse. 


\section{Entrainment of Touch and Posture}

Entrainment refers to an individual's chronobiological, physical, and behavioral relationship with their environment. Specifically, this refers to the coupling of two independent oscillatory systems in such a way that their periods of oscillation become related by virtue of phase alignment [12]. Contrasting the established idea of senses, Gibson considered separate anatomical units as perceptual systems [5]. In the present case, a joint yields spatial information, a skin-nerve conveys contact information, and in certain dynamic combinations, joint and skin-nerve yield synchronization or entrainment specifying information about the layout of external surfaces during locomotion.

Behavioral dynamics in a consistent approach have been proposed to account for the dynamics of perception and action [14]. This approach followed Gibson's idea that rather than being localized in an internal (or external) structure, control is distributed over the agent-environment system, in the present case, the user-artifact-surface system. Therefore, Warren's behavioral dynamics argues for a one-to-one correspondence between the internal structure IKS (Instantaneous knee screw) [15], constituted by the internal forces formed by the distal end of the femur and the proximal end of the tibia and the external structure, represented by the ground reaction forces (GRFs) on foot [16]

To test such an ecological approach to perception and action during the stance phase of a gait, we compared previously published experimental data sets [17] with our predicted datasets [18] in terms of medial and lateral contact forces. Available data included limb motion capture, fluoroscopy images, GRFs, electromyographical readings determining muscle forces, as well as medial and lateral knee contact forces derived from GRFs. Data were collected from an adult male with a right knee reconstruction $(65 \mathrm{~kg}$ mass and $1.7 \mathrm{~m}$ height).

In this study, the IKS was determined by a linear combination of two separate instantaneous screw axes of the shank (S) and thigh (T) Figure 1(a). Let the motion of (S, T) referred to their respective axes, the instantaneous shank axis (ISS) and the instantaneous thigh axis (ITS) respectively Figure 1(a); the motion of the shank referred to the same system of coordinates as the thigh, is obtained by the transformation of coordinates. The motion of the shank then takes the form Figure 1(b). This follows from the well-known result that a pair of $(\mathrm{S}, \mathrm{T})$ have the IKS in common velocity [19]. Then, the motion of the shank at the IKS has to be momentarily at rest and stay within the thigh. We can introduce a reference system that moves with the thigh, and we can observe the shank in that system. The criterion for the equilibrium of an arbitrary system of forces at the given knee is that the total virtual work of all forces vanishes [20]. This criterion involves virtual, not actual displacements, and at that instant, the actual motions of the $\mathrm{T}$ and $\mathrm{S}$ enter into account as the invariant ISS and ITS Figure 1(a).

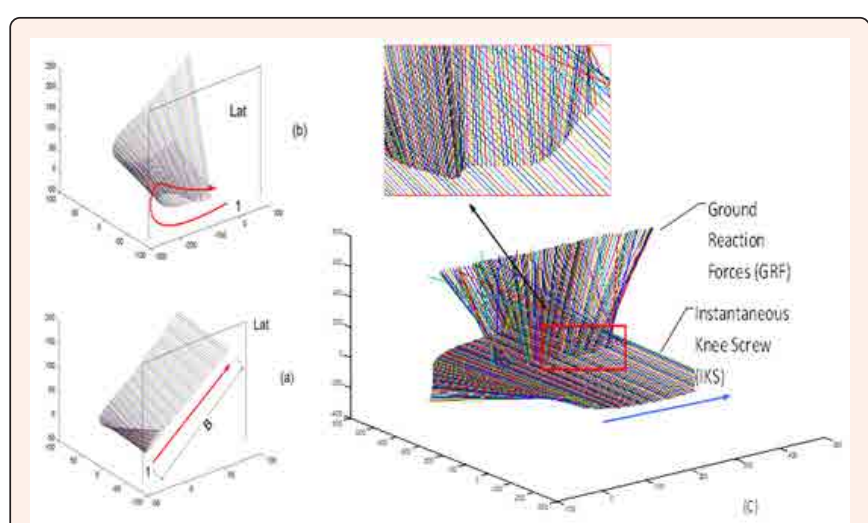

Figure 1: Figure 1(a,b): An actual displacement by performatory actions on the instantaneous screw axes (ISA) from the posteromedial side of the shank (a) and the thigh(b). The endpoints of axes are at the intersections with medial and lateral sagittal planes, which are located $-60 \mathrm{~mm}$ to $60 \mathrm{~mm}$ off the origin of the global frame. The first axis, indicated by 1 , represents the first step of IKS. The arrow indicates where the subsequent axes are migrated at every subsequent $0.05 \mathrm{sec}$ time increment (units in $\mathrm{mm}$ ); Figure 1(c): A virtual displacement by exploratory actions of perception and action during the stance phase of gait entrain the knee joint rotation with the touch pattern (GRF) of the foot. The invariant knee-manifolds demonstrates that an affordance for postural stability is measured relative to the posture of the patient, as represented by the entrainment of the GRF with the IKS at any point in the gait pathway.
Since the virtual displacement, the variation of the IKS, involves a possible but purely mathematical experiment, it can be applied at a certain definite time (even if such a displacement would involve physiologically infinite velocities). As an affordance of the knee for a patient, however, the IKS's have to be measured relative to the patient. They have unity relative to the posture and touch of the patient being considered [11]. Coupling introduces a constraint on the behavior of each limb. The motion of the shank is no longer completely independent of the phase and velocity of the thigh. In relative coordination, there is a tensegrity structure between the intrinsic dynamic structure of each of the two systems and the coupling force that links them. Behavioral dynamics control laws indicate that the entrainment or coordination of the shank and thigh $(\mathrm{S}, \mathrm{T})$ follows the same physical laws as the entrainment between the knee and ground (IKS, GRF). In order to tease out the implications of this claim, it will be necessary to introduce and clarify the notions of both affordance and entrainment. Coupling of $(\mathrm{S}, \mathrm{T})$ introduces a constraint on the behavior of each limb. The motion of one limb is no longer completely independent of the phase and velocity of the other. This very important characteristic of coupled systems generally has as a consequence that the resulting composite system is effectively of a lower dimension than the aggregate of the components [12]. Therefore, the cross-ratio [21] of the ordered pair (IKS, GRF) with respect to the ordered pair $(\mathrm{S}, \mathrm{T})$ is

$$
\{(I K S, G R F) ;(S, T)\}=-1
$$

In particular, if $\{(I K S, G R F) ;(S, T)\}=-1$, then $(I K S, G R F)$ divide a pair of (ISS, ITS) harmonically [22]. The fundamental hypothesis of this work is that affordances of the knee create selection pressure on the behavior of individual limbs, as perceived by its invariant, ISS, and ITS; hence is regulated with respect to the affordances of the environment for a given patient. One of most profound is that a pair of invariant (ISS, ITS) can be so selected with reference to the other pair of invariant belonging to the knee system (IKS, GRF) that the IKS nearly coincides with a reciprocal screw of the GRF, as indicated in a magnified inset image in Figure 1(b). The motion of one limb is no longer completely independent of the phase and velocity of the other. The IKS is perceived as an affordance for the entrainment of movement of $(\mathrm{S}, \mathrm{T})$.

A perceptual system of the knee can come to equilibrium since twists of amplitudes $S$ and T neutralize. We thus see that the two kinds of action: actual motion at the knee joint $(\mathrm{S}, \mathrm{T})$, can be selected with reference to the virtual work function of (IKS, GRF) as also categorized as the performatory and exploratory action during human walking [11]. Active organisms of the knee that can obtain and utilize information about persisting properties of their tissue environments in which the grafts are to be located will have a definite advantage over organisms that cannot do this. When the variations in the ground contact (magnitudes and direction) were shown along with the variations of knee movement in terms of IKS, an invariant was determined uniquely by the two corresponding pairs, see equation (1) (Figure 1(b)).

For a given IKS (when an observer perceives the affordance of the surface) and the location of the center of pressure (COP) on the axis of the GRF is known, then the GRF vector is limited to a plane in the screw system of the first order [23] (Figure 1(a)). The muscle synergy $\eta$ and GRF $\varphi$ are then compounded into an invariant, limited to the plane of the COP in reciprocity with the IKS. This theorem was originally proposed by Möbius, who showed that forces from six lines could be equilibrated, and also, if five of the lines are given along with a point on the sixth line, then the sixth line is limited to a polar plane [19].

Thus, the affordance of the knee has the potential to diagnose pathologies. The last decade has seen a paradigm shift in the measurement of clinical outcomes, with an increasing focus on the user's perspective, PROMs. Many clinicians, though, are less confident in self-reported PROMs, than in 'objective measurements' [10]. Recent studies identified several sensations, activities, and psychological factors such as feelings of instability and knee-related fears that make the patients aware of their artificial knee joint [24]. They concluded that joint awareness might work as an overarching parameter. This is aligned with Gibson's statement that an affordance cuts across the dichotomy of subjective-objective and helps us to understand its inadequacy [11]. Affordances have to be designed in relation to the uniqueness of each patient, and thus posture and movement need to be measured in terms of a specific patient-environment system, not in patientcentered terms.

\section{Acknowledgment}

The authors acknowledge the support from The MIT-Peru UTEC Seed Fund awarding the project titled "Development of Proper Tunnel Syndrome Placement Device to Avoid Impingement." The experimental data used for validation were provided by the "Grand Challenge Competition to Predict in Vivo Knee Loads" as part of the Symbiosis 
project funded by the US National Institutes of Health via the NIH Roadmap for Medical Research (Grant \# U54 GM072970).

\section{References}

1. Howell S (1998) Principles for placing the tibial tunnel and avoiding roof impingement during reconstruction of a torn anterior cruciate ligament. Knee Surg Sports Traumatol Arthrosc 6: S49-S55.

2. Behrend H, K Giesinger, V Zdravkovic, J M Giesinger (2017) Validating the forgotten joint score-12 in patients after ACL reconstruction. The Knee 24: 768-774.

3. Karlsson, J, M T Hirschmann, R Becker, V Musahl (2015) Individualized ACL surgery. Knee Surg Sports Traumatol Arthrosc 23: 2143-2144.

4. Johansson H, P Sjölander, P Sojka (1991) Receptors in the knee joint ligaments and their role in the biomechanics of the joint. Crit Rev Biomed Eng 18: 341-368.

5. Gibson J J (1966) The senses considered as perceptual systems. Boston, Houghton.

6. Niama Natta D D, E Thienpont, A Bredin, G Salaun, C Detrembleur (2019) Rasch analysis of the Forgotten Joint Score in patients undergoing knee arthroplasty. Knee Surg Sports Traumatol Arthrosc 27: 1984-1991.

7. Kinnaman J E S, A D Farrell, S W Bisconer (2006) Evaluation of the Computerized Assessment System for Psychotherapy Evaluation and Research (CASPER) as a Measure of Treatment Effectiveness with Psychiatric Inpatients. Assessment 13: 154-167.

8. Rolfson O, K Eresian Chenok, E Bohm, A Lübbeke, et al. (2016) Patient-reported outcome measures in arthroplasty registries. Acta orthopaedica 87: 3-8.

9. Behrend H, K Giesinger, J M Giesinger, M S Kuster (2012) The "Forgotten Joint" as the Ultimate Goal in Joint Arthroplasty. J Arthroplasty 27: 430-436.

10. Hamilton D F, J M Giesinger, K Giesinger (2017) It is merely subjective opinion that patient-reported outcome measures are not objective tools. Bone \& joint research Bone Joint Res 6: 665-666.

11. Gibson J J (1979) The ecological approach to visual perception, Houghton Mifflin.
12. Cummins F (2009) Rhythm as an affordance for the entrainment of movement Phonetica 66: 15-28.

13. Reed E S (1996) Encountering the world: Toward an ecological psychology, Oxford University Press.

14. Warren W H (2006) The dynamics of percept ion and action. Psychol Rev 113: 358-389.

15. Kim W, D Araujo, S S Kohles, S G Kim, H H Alvarez Sanchez (2020) AffordanceBased Surgical Design Methods Considering Biomechanical Artifacts. Ecological Psychology 33: 57-71.

16. Beer F P (2010) Vector Mechanics for Engineers: Statics and dynamics, McGrawHill Companies.

17. Fregly B J, T F Besier, D G Lloyd, S L Delp, S A Banks, et al. (2012) Grand challenge competition to predict in vivo knee loads. J Orthop Res 30: 503-513.

18. Kim W, A P Veloso, D Araújo, V Vleck, F João (2013) An informational framework to predict reaction of constraints using a reciprocally connected knee model. Comput Methods Biomech Biomed Engin 18: 78-89.

19. Ball R (1900) A treatise on the theory of screws, Cambridge University Press.

20. Lanczos C (2012) The variational principles of mechanics, Courier Corporation, US pp 418.

21. Semple J G, G T Kneebone (1960) Algebraic Projective Geometry, Clarendon Press, UK.

22. Courant R, H Robbins (1941) what is Mathematics? An elementary approach to ideas and methods. London, New York etc, Oxford University press.

23. Kim W, A P Veloso, V E Vleck, C Andrade, S S Kohles (2013) The stationary configuration of the knee. J Am Podiatr Med Assoc 103: 126-135.

24. Loth F L, M C Liebensteiner, J M Giesinger, K Giesinger, H R Bliem (2018) What makes patients aware of their artificial knee joint?" BMC Musculoskeletal Disorders 19: 5 